\title{
THE CONTRIBUTION OF EARTHQUAKES TO THE DEFORMATION OF ZAGROS TECTONIC PROVINCE
}

\author{
M. A. Sharifi ${ }^{1}$, A. Bahroudi ${ }^{2}$, S. Mafi ${ }^{3 . *}$ \\ ${ }^{1}$ Research Institute of Geoinformation Technology, School of Surveying and Geospatial Engineering, Faculty of Engineering, \\ University of Tehran, Tehran, Iran - sharifi@ut.ac.ir \\ ${ }^{2}$ Exploration Department, School of Mining Engineering, Faculty of Engineering, University of Tehran, Tehran, Iran - \\ bahroudi@ut.ac.ir \\ ${ }^{3}$ School of Surveying and Geospatial Engineering, Faculty of Engineering, University of Tehran, Tehran, Iran - \\ saleh.mafi@gmail.com
}

KEY WORDS: Seismic moment rate, Geodetic moment rate, Shear strain rate, Dilative strain rate, Earthquake magnitude, Velocity vectors of geodynamic network,

\begin{abstract}
:
In this study, we investigate the contribution of earthquakes to the deformation of Zagros province and compare the seismicity and the density of earthquakes in different parts of the province. The mathematics used in this research is based on calculations of moment rates. The seismic moment rate is the average amount of seismic energy releases from the tectonic province in each year. The geodetic moment rate is the average amount of energy which is consumed every year to make deformation in Zagros. The ratio of these two moment rates expresses the contribution of earthquakes in making deformation in Zagros province. According to the calculations, this ratio is estimated to be $13.06 \%$. Along with the information obtained from the moment rates, we can also obtain the shear and the dilative strain rates from the strain rate tensors, which show the volumetric changes and the deformation rate in different parts of the Zagros, respectively. The data used in this study include the focal coordinates of the Zagros earthquakes with their magnitude and the velocity vectors of the Zagros geodynamic network, which are used to calculate the seismic and the geodetic moment rates.
\end{abstract}

\section{INTRODUCTION}

The Zagros fold-thrust belt is an orogenic belt along the NWSE that stretches about $2000 \mathrm{~km}$ from Turkey to the Strait of Hormuz in the southwest of Iran (Berberian, 1995). This belt is limited to the north by the main Zagros fault, which is referred to as connection of Neo-Tethys ocean. Recent tectonic activities in this area are the result of convergence of the continental plates of Arabia and Eurasian from the late Cretaceous/early Miocene. This belt, which is the youngest continental conjunction zone on the planet, is a key area for studying the processes that occurred at the early stages of the formation of convergent regions (Jackson et al. 1981).

In general, the orogeny phenomenon and the other aspects of deformation created on the earth's surface are responses to the stresses imposed to different parts of the earth's crust. Earthquakes, as one of the important factors of deformation, are the result of the accumulation of elastic energy in the earth's crust, and the greater amount of energy accumulated in the crust, the larger earthquake will happen. Investigation of the focal mechanisms of Zagros earthquakes indicates shortening and thickening in the main fault and the reverse faults of the Zagros which are responsible for the deformation in this area (Mostafazadeh et al. 2000; Talebian and Jackson, 2004). Based on the slip rate and the attitude of deformation obtained from GPS observations, Zagros is divided into two parts: the North Zagros and the Central Zagros. The right lateral strike slip Kazerun fault is considered as the border between the North and the Central Zagros (Baker et al. 1993; Vernant et al. 2004). In the North Zagros, the deformation is decomposed into a (3-6) mm/Year shortening component perpendicular to the
Zagros strike and a $(4-6) \mathrm{mm} /$ Year strike slip component parallel to the Zagros. In the Central Zagros, the deformation has a $8 \pm 2 \mathrm{~mm} /$ Year shortening component that is perpendicular to the Zagros strike (Walpersdorf et al. 2006). The larger part of the deformation is observed in the southern part of the Zagros, while seismicity is distributed all over the region.

By combining the information obtained from geological and tectonic studies and seismic data, we can determine some areas on a geological map which have different seismic power and distinguish from the adjacent areas. These areas are called tectonic provinces.

In this study, we investigate the contribution of earthquakes in making deformation in Zagros and interpret the deformation rate in different regions of the tectonic province by using the strain rate tensor. These calculations are based on the seismic and geodetic moment rates. The seismic moment rate of the Zagros represents the average amount of seismic energy releases every year from the tectonic province. The geodetic moment rate is also the average amount of energy that each year is spent on creating the deformation in Zagros. The ratio of these two moment rates indicates the contribution of earthquakes in making deformation in Zagros. The shear strain rate and the dilative strain rate can be obtained by calculating the strain rate tensor. Each of these parameters describes a different description of the deformation rate in Zagros. We can conclude new information about the seismic activity of the Zagros faults by interpreting the strain rate components and the density of the earthquakes in Zagros province. 


\section{PROPOSED METHOD}

In this research, we use the seismic data to calculate the seismic moment rate. These data are provided by the International Institute of Earthquake Engineering and Seismology (IIEES).

Mirzaei et al (1998) delineated five major tectonic provinces in Iran based on all available geophysical, geological, tectonic and earthquake data (Figure 1). Earthquake magnitudes are expressed in different scales in the seismic catalogues and must be converted to moment magnitude $\left(M_{W}\right)$. The conversion equations of the magnitude scales $M_{W}-M_{S}$ and $M_{W}-m_{b}$ are as follows (Scordilis, 2006):

$$
\begin{array}{ll}
M_{W}=0.67( \pm 0.005) M_{S}+2.07( \pm 0.03) & 3.0 \leq M_{S} \leq 6.1 \\
M_{W}=0.99( \pm 0.020) M_{S}+0.08( \pm 0.13) & 6.2 \leq M_{S} \leq 8.2 \\
M_{W}=0.85( \pm 0.040) m_{b}+1.03( \pm 0.23) & 3.5 \leq m_{b} \leq 6.2
\end{array}
$$

$M_{W}-M_{L}$ and $M_{W}-M_{D}$ relations have been calculated as follows respectively (Mousavi-Bafrouei et al. 2014 ; Yenier et al. 2008):

$$
\begin{array}{ll}
M_{W}=0.81( \pm 0.045) M_{L}+1.098( \pm 0.22) & 3.7 \leq M_{L} \leq 6.4 \\
M_{W}=0.764 M_{D}+1.379 & 3.7 \leq M_{D} \leq 6.0
\end{array}
$$

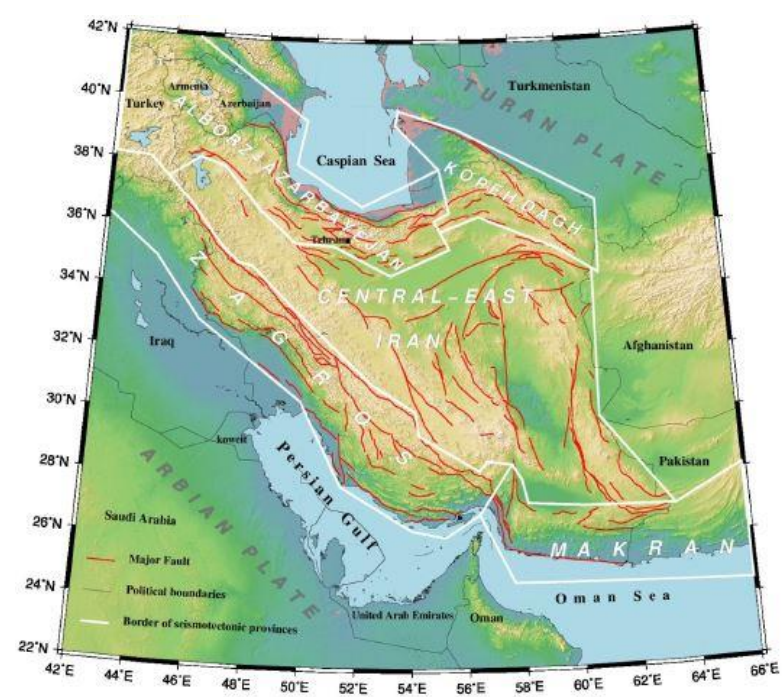

Figure 1. Major tectonic provinces of Iran developed by Mirzaei et al (1998). The red lines depict the major active faults developed by Hessami et al (2003).

The scalar seismic moment $\left(M_{0}\right)$ is calculated using the following equation (Kanamori, 1977):

$$
M_{0}=10^{\left(1.5 M_{W}+9\right)}
$$

This parameter describes the amount of seismic energy releases at the time of earthquake. The total seismic energy of the Zagros earthquakes in each year indicates the seismic moment of the Zagros province (Figure 4 ).
The cumulative seismic moments are calculated by using the seismic moments. A degree one polynomial is fitted to the cumulative moments and the slope of the fitted line is considered as the seismic moment rate (Figure 5).

Ward (1998) used the following equation to calculate the geodetic moment rate:

$$
\dot{M}_{0}^{W}=2 \mu H A\left|\dot{\lambda}_{\max }\right|
$$

Where $\mu$ is the shear modulus of crust which is considered to be $3 * 10^{10} \mathrm{~N} / \mathrm{m}^{2}, H$ is the thickness of the seismogenic layer, $A$ is the network triangle area and $\left|\lambda_{\max }\right|$ is the absolute of maximum eigenvalue obtained from the $2 \mathrm{D}$ strain rate tensor.

The seismogenic layer is that part of the earth's crust whose deformation is elastic, and the major fractures caused by the earthquakes occur in this part. Considering the effect of the systematic errors in the focal coordinates of the earthquakes (including the error of the focal depth and the epicenter location), we don't use all of these coordinates to calculate the seismogenic thickness. Results of waveform modeling and microearthquake studies reveal that the majority of earthquakes in Zagros occur in depths of $8 \sim 15 \mathrm{~km}$ (Mirzaei et al. 1997). In order to decrease the effects of systematic errors, we use the coordinates whose focal depths are less than $20 \mathrm{~km}$. The thickness of the seismogenic layer is calculated at the centroids of the network triangles (Figure 6) by using a weighted average of the focal depths. We use the Delaunay method to triangulate the geodynamic network of the Zagros. The following equations describe how to calculate this parameter.

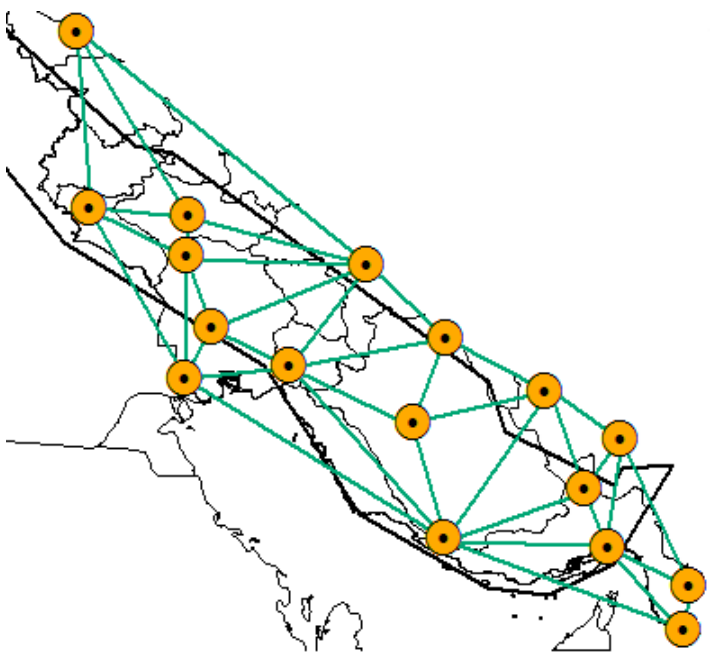

Figure 2. Triangulation of the Zagros geodynamic network using the Delaunay method.

$$
r_{H y p}^{C}=\sqrt{\left(x^{C}-x_{H y p}\right)^{2}+\left(y^{C}-y_{H y p}\right)^{2}+\left(z^{C}-z_{H y p}\right)^{2}}
$$

$$
w_{i}^{j}=\frac{1}{\left(r_{\text {Hyp }}^{C}\right)^{m}}
$$




$$
H_{S}^{i}=\frac{\sum_{j=1}^{N} w_{i}^{j} D_{j}}{\sum_{j=1}^{N} w_{i}^{j}}
$$

Where $D_{j}$ is the focal depth of the $\mathrm{j}^{\text {th }}$ earthquake, $r_{H y p}^{C}$ is the Euclidean distance between the centroid of a network triangle and the hypocenter of an earthquake, $N$ is the total number of Zagros earthquakes, $H_{S}^{i}$ is the estimation of the seismogenic thickness in the centroid of each triangle and $m$ is the power of distance which its value is determined empirically. Here, $m$ is considered to be 2 .

The coordinates of the geodynamic network stations are expressed in the geodetic coordinates $(\phi, \lambda, h)$. Where $\phi$ is the geodetic latitude, $\lambda$ is the geodetic longitude and $h$ is called the geodetic height. Table (1) and Table (2) include the geodetic coordinates of the GPS stations and the components of the velocity vectors. The Cartesian coordinates of the GPS stations $(x, y, z)$ are calculated as follows (Jekeli, 2006):

$x^{\text {sta }}=\left(\frac{a}{\sqrt{1-e^{2} \sin ^{2}(\phi)}}+h\right) \cos (\phi) \cos (\lambda)$

$y^{\text {sta }}=\left(\frac{a}{\sqrt{1-e^{2} \sin ^{2}(\phi)}}+h\right) \cos (\phi) \sin (\lambda)$

$z^{s t a}=\left(\frac{a\left(1-e^{2}\right)}{\sqrt{1-e^{2} \sin ^{2}(\phi)}}+h\right) \sin (\phi)$

Where $a=6378137 \mathrm{~m}$ and $e=0.0818$ are the semi-major axis and the eccentricity of the WGS84 ellipsoid, respectively.

By calculating the Cartesian coordinates of the permanent stations, the Cartesian coordinates of the centroids in equation (8) are calculated as follows:

$$
x^{C}=\frac{\sum_{i=1}^{3} x_{i}^{s t a}}{3}
$$

$y^{C}=\frac{\sum_{i=1}^{3} y_{i}^{s t a}}{3}$

$$
z^{C}=\frac{\sum_{i=1}^{3} z_{i}^{\text {sta }}}{3}
$$

The focal coordinates of the earthquakes' hypocenters are expressed in geodetic latitude, geodetic longitude and focal depth $(D)$ in the seismic catalogues. In order to calculate the Cartesian coordinates of the hypocenters $\left(x_{H y p}, y_{H y p}, z_{H y p}\right)$ in equation (8), we need to convert the focal depths to the geodetic heights $(h)$. This conversion is carried out by the SRTM heights. Considering the epicenter of each earthquake, we can find an SRTM cell of 4 points around the epicenter which are the nearest points to the epicenter (Figure 3).

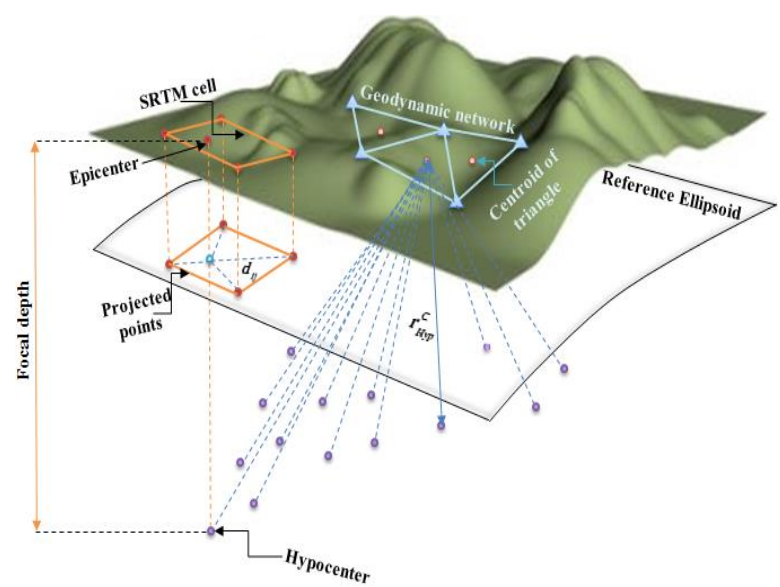

Figure 3. Calculation of seismogenic thickness. The red dots are the vertices of the SRTM cell around the earthquake epicenter. The geodetic height of the hypocenter is calculated by using the geodetic heights of the SRTM points and the focal depth.

The geodetic height of the epicenter point is calculated by using a weighted average as follows:

$h_{E p i}=\frac{\sum_{i=1}^{4} h^{i} w_{E p i}^{i}}{\sum_{i=1}^{4} w_{E p i}^{i}}$

$w_{E p i}^{i}=\frac{1}{d_{j}^{i}}$

Where $h^{i}$ is the geodetic height of the $\mathrm{i}^{\text {th }}$ vertex of the SRTM cell around the epicenter point and $d_{j}^{i}$ is the Euclidean distance between the projected $j^{\text {th }}$ Epicenter and the projected $i^{\text {th }}$ vertex on the reference ellipsoid. For projecting the points on the reference ellipsoid, it is enough to consider the geodetic heights as zero. The geodetic latitude and longitude of the points are also known. So, we can determine the Cartesian coordinates of the projected points using the equations (11), (12) and (13). By calculating the geodetic height of the epicenter, the geodetic height of the hypocenter will be calculated simply:

$h_{H y p}=h_{E p i}-D$

Where $D$ is the focal depth of the hypocenter. Now, the Cartesian coordinates of the hypocenter can be determined and the Euclidean distance $r_{H y p}^{C}$ in equation (9) is calculated.

To calculate the eigenvalues of the $2 \mathrm{D}$ strain rate tensor, we use the finite element method. The elements of the tensor are calculated at the centroids of the network triangles (Figure 2). Strain rate tensor is a symmetric tensor, so it has 6 independent elements. The following equations show how to calculate these elements:

$u_{i}=\left(\frac{\partial u}{\partial x}\right)_{j} x_{i}+\left(\frac{\partial u}{\partial y}\right)_{j} y_{i}+a_{j}+r_{j i}$ 
$v_{i}=\left(\frac{\partial v}{\partial x}\right)_{j} x_{i}+\left(\frac{\partial v}{\partial y}\right)_{j} y_{i}+b_{j}+r_{j i}$

$u_{i}=\left(X_{i}^{(2)}-X_{i}^{(1)}\right) / \Delta t$

$v_{i}=\left(Y_{i}^{(2)}-Y_{i}^{(1)}\right) / \Delta t$

$x_{i}=X_{i}-x^{C}$

$y_{i}=Y_{i}-y^{C}$

Where $u_{i}$ and $v_{i}$ are the components of the velocity vectors available in the Table (2), $\left(X_{i}, Y_{i}\right)$ are the Cartesian coordinates of the triangle vertex and $\left(x^{C}, y^{C}\right)$ are the Cartesian coordinates of the triangle centroid. $\left(\frac{\partial u}{\partial x}\right)_{j},\left(\frac{\partial u}{\partial y}\right)_{j},\left(\frac{\partial v}{\partial x}\right)_{j},\left(\frac{\partial v}{\partial y}\right)_{j}, a_{j}$ and $b_{j}$ are the unknown parameters in equations (20) and (21). By calculating these parameters, the strain rate tensor is determined as follows:

$$
\dot{\varepsilon}=\left[\begin{array}{cc}
\left(\frac{\partial u}{\partial x}\right)_{j} & \frac{1}{2}\left(\left(\frac{\partial u}{\partial y}\right)_{j}+\left(\frac{\partial v}{\partial x}\right)_{j}\right) \\
\frac{1}{2}\left(\left(\frac{\partial u}{\partial y}\right)_{j}+\left(\frac{\partial v}{\partial x}\right)_{j}\right) & \left(\frac{\partial v}{\partial y}\right)_{j}
\end{array}\right]
$$

The absolute of maximum eigenvalue for this tensor is used to calculate the geodetic moment rate in equation (7).

We can find the following relationships between the eigenvalues of the strain rate tensor.

$\dot{\lambda}_{\min }=\frac{\left(\dot{\varepsilon}_{11}+\dot{\varepsilon}_{22}\right)-\sqrt{\left(\dot{\varepsilon}_{11}\right)^{2}+\left(\dot{\varepsilon}_{22}\right)^{2}-2 \dot{\varepsilon}_{11} \dot{\varepsilon}_{22}+4\left(\dot{\varepsilon}_{12}\right)^{2}}}{2}$

$\dot{\lambda}_{\max }=\frac{\left(\dot{\varepsilon}_{11}+\dot{\varepsilon}_{22}\right)+\sqrt{\left(\dot{\varepsilon}_{11}\right)^{2}+\left(\dot{\varepsilon}_{22}\right)^{2}-2 \dot{\varepsilon}_{11} \dot{\varepsilon}_{22}+4\left(\dot{\varepsilon}_{12}\right)^{2}}}{2}$

$\dot{\Delta}_{2 D}=\dot{\lambda}_{\max }+\dot{\lambda}_{\min }=\dot{\varepsilon}_{11}+\dot{\varepsilon}_{22}$

$\dot{\Delta}_{2 D}$ is the dilative strain rate parameter derived from the 2D strain rate tensor. We can find a geometric concept for the dilative strain rate by following definition:

$\dot{\Delta}_{2 D}=\frac{\dot{\Delta S}}{S}=\frac{\dot{\Delta x}}{x}+\frac{\dot{\Delta y}}{y}=\dot{\varepsilon}_{11}+\dot{\varepsilon}_{22}$

The 2D shear strain rate is defined by the following equation:

$$
\begin{aligned}
& \dot{\gamma}_{2 D}=\dot{\lambda}_{\max }-\dot{\lambda}_{\min }=\sqrt{\left(\dot{\varepsilon}_{11}-\dot{\varepsilon}_{22}\right)^{2}+4\left(\dot{\varepsilon}_{12}\right)^{2}} \\
& \dot{\gamma}_{2 D}=\sqrt{\left(\frac{\dot{\Delta x}}{x}-\frac{\dot{\Delta y}}{y}\right)^{2}+4\left(\frac{1}{2}\left(\frac{\dot{\Delta x}}{y}+\frac{\dot{\Delta y}}{x}\right)\right)^{2}} \\
& \dot{\gamma}_{2 D}=\sqrt{\frac{\dot{\Delta x}^{2}+\dot{\Delta y y}^{2}}{x^{2}}+\frac{\dot{\Delta x}^{2}+\dot{\Delta y}^{2}}{y^{2}}}
\end{aligned}
$$

We can generalize the above equations to 3D format. In this case, we use all of the components of velocity vectors. The elements of the 3D strain rate tensor are calculated by using the finite difference method in each of the GPS stations.

$$
\begin{aligned}
& u_{i}=\left(\frac{\partial u}{\partial x}\right)_{j} x_{i}^{j}+\left(\frac{\partial u}{\partial y}\right)_{j} y_{i}^{j}+\left(\frac{\partial u}{\partial z}\right)_{j} z_{i}^{j}+a_{j}+r_{j i} \\
& v_{i}=\left(\frac{\partial v}{\partial x}\right)_{j} x_{i}^{j}+\left(\frac{\partial v}{\partial y}\right)_{j} y_{i}^{j}+\left(\frac{\partial v}{\partial z}\right)_{j} z_{i}^{j}+b_{j}+r_{j i} \\
& w_{i}=\left(\frac{\partial w}{\partial x}\right)_{j} x_{i}^{j}+\left(\frac{\partial w}{\partial y}\right)_{j} y_{i}^{j}+\left(\frac{\partial w}{\partial z}\right)_{j} z_{i}^{j}+c_{j}+r_{j i}
\end{aligned}
$$

Where $u_{i}, v_{i}$ and $w_{i}$ are the components of the velocity vectors. $x_{i}^{j}, y_{i}^{j}$ and $z_{i}^{j}$ are defined as follows:

$$
\begin{gathered}
x_{i}^{j}=X_{i}-X_{j} \\
y_{i}^{j}=Y_{i}-Y_{j} \\
z_{i}^{j}=Z_{i}-Z_{j}
\end{gathered}
$$

Where $\left(X_{j}, Y_{j}, Z_{j}\right)$ are the coordinates of the station that we're going to calculate its tensor elements. The elements of the 3D strain rate tensor, the 3D dilative and shear strain rates calculate as follows:

$$
\begin{aligned}
& \dot{\varepsilon}_{k l}=\frac{1}{2}\left(\left(\frac{\partial u_{k}}{\partial x_{l}}\right)_{j}+\left(\frac{\partial u_{l}}{\partial x_{k}}\right)_{j}\right) \\
& k=1,2,3 \quad l=1,2,3 \\
& u_{1}=u \quad u_{2}=v \quad u_{3}=w \\
& x_{1}=x \quad x_{2}=y \quad x_{3}=z
\end{aligned}
$$$$
\dot{\Delta}_{3 D}=\frac{\dot{\Delta V}}{V}=\frac{\dot{\Delta x}}{x}+\frac{\dot{\Delta y}}{y}+\frac{\dot{\Delta z}}{z}=\dot{\varepsilon}_{11}+\dot{\varepsilon}_{22}+\dot{\varepsilon}_{33}
$$

$$
\dot{\gamma}_{3 D}=\sqrt{\frac{\dot{\Delta x}^{2}+\dot{\Delta y}^{2}+\dot{\Delta z}^{2}}{x^{2}}+\frac{\dot{\Delta x}^{2}+\dot{\Delta y}^{2}+\dot{\Delta z}^{2}}{y^{2}}+\frac{\dot{\Delta x}^{2}+\dot{\Delta y}^{2}+\dot{\Delta z}^{2}}{z^{2}}}
$$




$$
\dot{\gamma}_{3 D}=\sqrt{\sum_{i=1}^{3} \dot{\varepsilon}_{i i}^{2}+4 \sum_{\substack{i, j=1 \\ i \neq j, j>i}}^{3} \dot{\varepsilon}_{i j}^{2}-2 \sum_{\substack{i, j=1 \\ i \neq j, j>i}}^{3} \dot{\varepsilon}_{i i} \dot{\varepsilon}_{j j}}
$$

Table 1. The geodetic coordinates of the Zagros geodynamic stations

\begin{tabular}{|c|c|c|c|}
\hline Station & lat $(\mathrm{deg})$ & lon $(\mathrm{deg})$ & $\mathrm{h}(\mathrm{m})$ \\
\hline BRBS & 27.2072 & 56.3206 & 23.6423 \\
\hline JASC & 25.6375 & 57.7699 & 1.1230 \\
\hline LAMD & 27.3637 & 53.2034 & 401.9124 \\
\hline SHRZ & 29.5444 & 52.6026 & 1493.326 \\
\hline BEBN & 30.6057 & 50.2169 & 316.8581 \\
\hline BAFT & 29.2392 & 56.58 & 2286.743 \\
\hline ABRK & 31.1205 & 53.2265 & 1537.25 \\
\hline SFHN & 32.5177 & 51.7061 & 1550.34 \\
\hline AHVZ & 31.3439 & 48.7444 & 19.7275 \\
\hline ABDN & 30.3778 & 48.2135 & 1.5774 \\
\hline ANGN & 26.4572 & 57.8973 & 739.3925 \\
\hline HOSN & 32.6699 & 48.2534 & 356.1099 \\
\hline KRAD & 33.4334 & 48.2787 & 1153.494 \\
\hline ILLM & 33.5887 & 46.3974 & 1331.247 \\
\hline SBAK & 30.1462 & 55.1075 & 1859.723 \\
\hline MIAN & 36.9083 & 46.1621 & 1309.429 \\
\hline HAJI & 28.2940 & 55.8946 & 947.7514 \\
\hline
\end{tabular}

Table 2 . The velocity vectors of the permanent stations

\begin{tabular}{|c|c|c|c|}
\hline Station & $\mathrm{V}_{\mathrm{E}}(\mathrm{mm} / \mathrm{y})$ & $\mathrm{V}_{\mathrm{N}}(\mathrm{mm} / \mathrm{y})$ & $\mathrm{V}_{\mathrm{U}}(\mathrm{mm} / \mathrm{y})$ \\
\hline BRBS & 32.54 & 30.20 & -1.21 \\
\hline JASC & 29.40 & 19.48 & 0.38 \\
\hline LAMD & 30.61 & 27.32 & -4.77 \\
\hline SHRZ & 26.73 & 22.21 & -4.46 \\
\hline BEBN & 24.36 & 27.03 & 0.02 \\
\hline BAFT & 28.92 & 21.47 & 0.86 \\
\hline ABRK & 27.09 & 22.30 & -5.88 \\
\hline SFHN & 26.78 & 21.31 & -0.3 \\
\hline AHVZ & 24.85 & 26.42 & -2.25 \\
\hline ABDN & 27.39 & 29.58 & -1.03 \\
\hline ANGN & 28.52 & 19.78 & -0.58 \\
\hline HOSN & 23.11 & 24.18 & 0.26 \\
\hline KRAD & 22.52 & 23.56 & -1.88 \\
\hline ILLM & 23.57 & 26.30 & 0.64 \\
\hline SBAK & 27.31 & 20.94 & -2.45 \\
\hline MIAN & 24.72 & 22.47 & -3.11 \\
\hline HAJI & 0.531 & -0.46 & 0.44 \\
\hline
\end{tabular}

It should be noted that the components of the velocity vectors have been expressed in the navigation coordinate system. In order to use these components, we need to convert them to the geodetic coordinate system. The following equation is used for performing the conversion (Drake, 2002).

$$
\left[\begin{array}{l}
V_{x} \\
V_{y} \\
V_{z}
\end{array}\right]=\left[\begin{array}{ccc}
-\sin (\lambda) & -\sin (\phi) \cos (\lambda) & \cos (\phi) \cos (\lambda) \\
\cos (\lambda) & -\sin (\phi) \sin (\lambda) & \cos (\phi) \sin (\lambda) \\
0 & \cos (\phi) & \sin (\phi)
\end{array}\right]\left[\begin{array}{c}
V_{E} \\
V_{N} \\
V_{U}
\end{array}\right]
$$

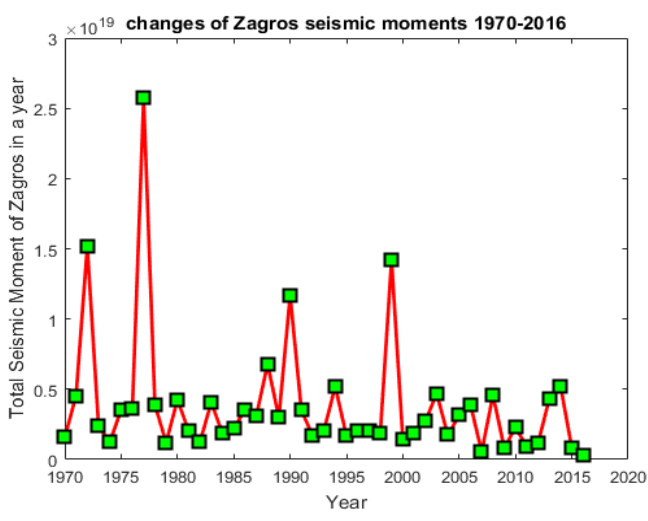

Figure 4. Changes of the seismic moment in Zagros from 1970 to 2016

The cumulative seismic moment in each year is calculated using the seismic moments of previous years. Figure (5) depicts the changes of the cumulative seismic moment in Zagros from 1970 to 2016 .

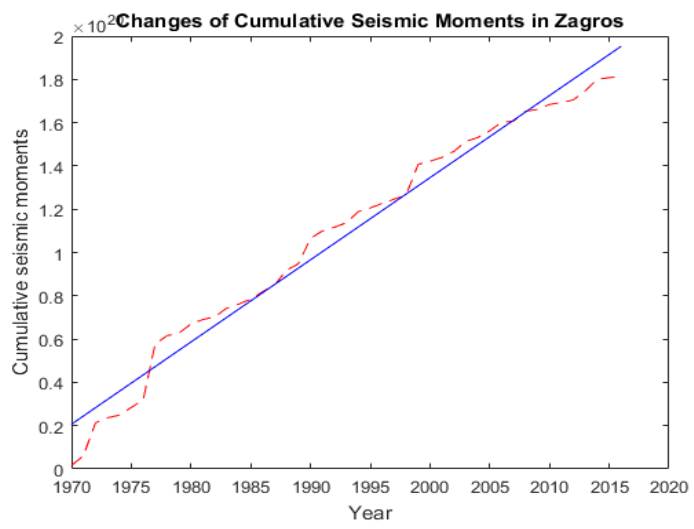

Figure 5. Changes of the cumulative seismic moment in Zagros from 1970 to 2016 and the seismic moment rate.

According to the calculations, the seismic moment rate is estimated to be $\dot{M}_{\text {Seismic }}=3.7977 * 10^{18} \mathrm{Nm} /$ Year which is the slope of the fitted line in Figure (5).

According to the calculations, the geodetic moment rate is estimated to be $\dot{M}_{\text {geodet } i c}=2.9082 * 10^{19} \mathrm{Nm} /$ Year . The contribution of the earthquakes to the Zagros deformation is determined by dividing the seismic moment rate to the geodetic moment rate which is about $13.06 \%$. In addition to calculation of the moment rates, the changes of the seismogenic thickness can also be depicted in different parts of the Zagros. Figure (6) depicts the changes of the seismogenic thickness in Zagros.

\section{RESULTS}

Figure (4) shows the changes of the seismic moment in Zagros from 1970 to 2016. 


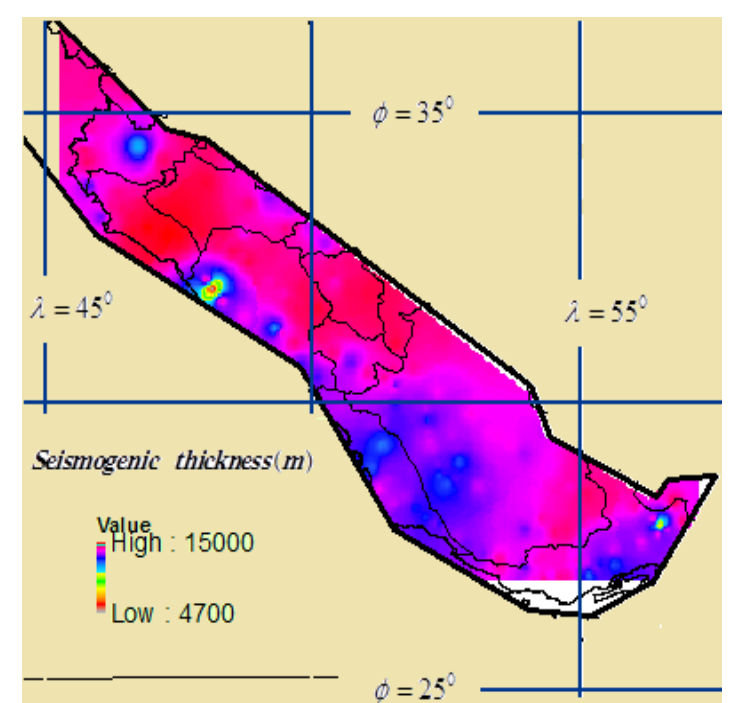

Figure 6. Changes of the seismogenic thickness in Zagros.

Figure (7) and Figure (8) describe the surface deformation in Zagros. It should be noted that these parameters are defined in the geodetic coordinate system. The $2 \mathrm{D}$ dilative strain rate $\left(\dot{\Delta}_{2 D}\right)$ shows the rate of the surface contraction or expansion in Zagros. The negative and the positive values mean contraction and expansion, respectively.

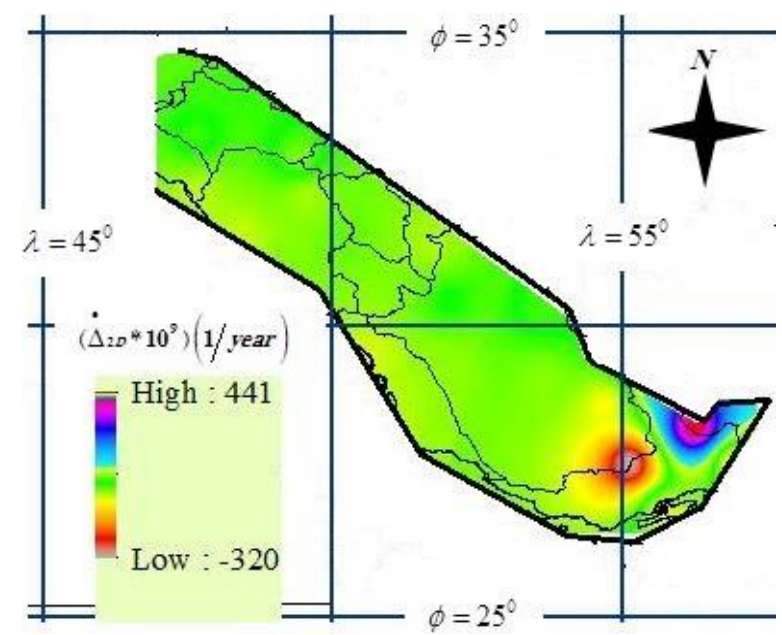

Figure 7. Changes of the $2 \mathrm{D}$ dilative strain rate in Zagros

The 2D shear strain rate $\left(\dot{\gamma}_{2 D}\right)$ shows the rate of absolute deformation in Zagros. This parameter does not contain the negative values.

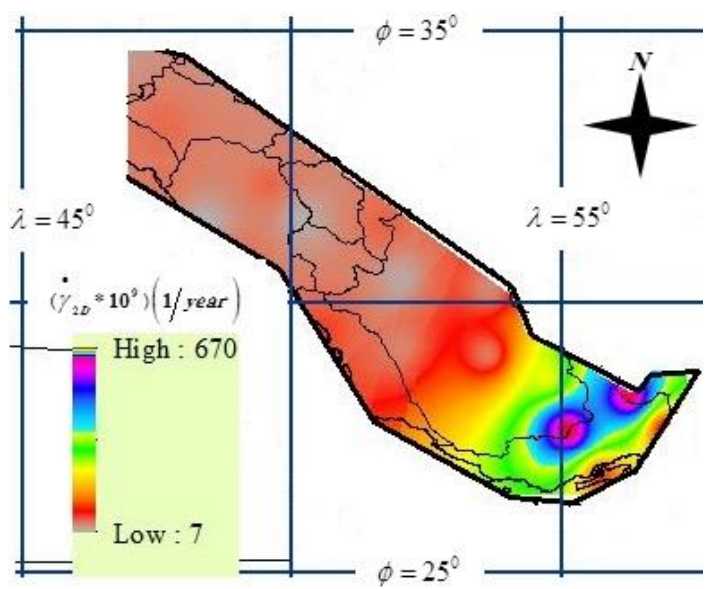

Figure 8. Changes of the 2D shear strain rate in Zagros

The 3D dilative and shear strain rates $\left(\dot{\Delta}_{3 D}\right.$ and $\left.\dot{\gamma}_{3 D}\right)$ also describe the $3 \mathrm{D}$ volumetric changes and the absolute $3 \mathrm{D}$ deformation in Zagros, respectively.

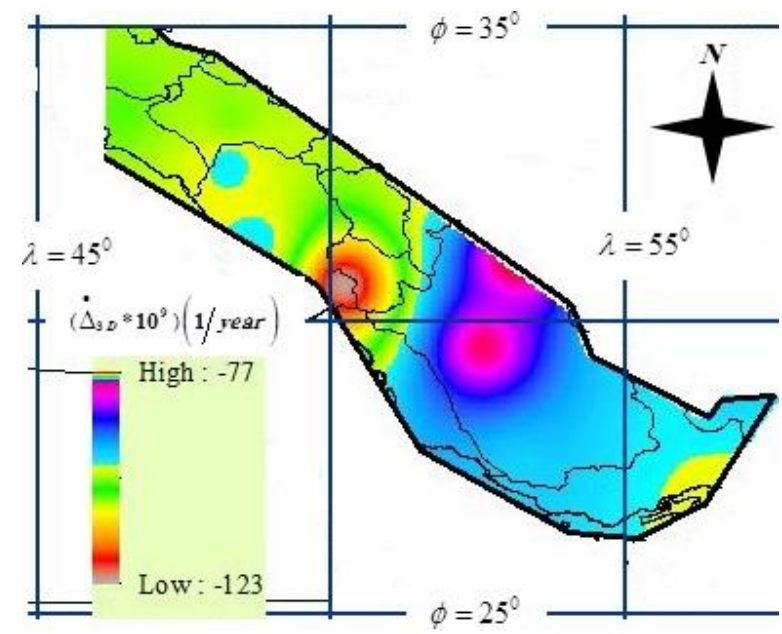

Figure 9. Changes of the 3D dilative strain rate in Zagros

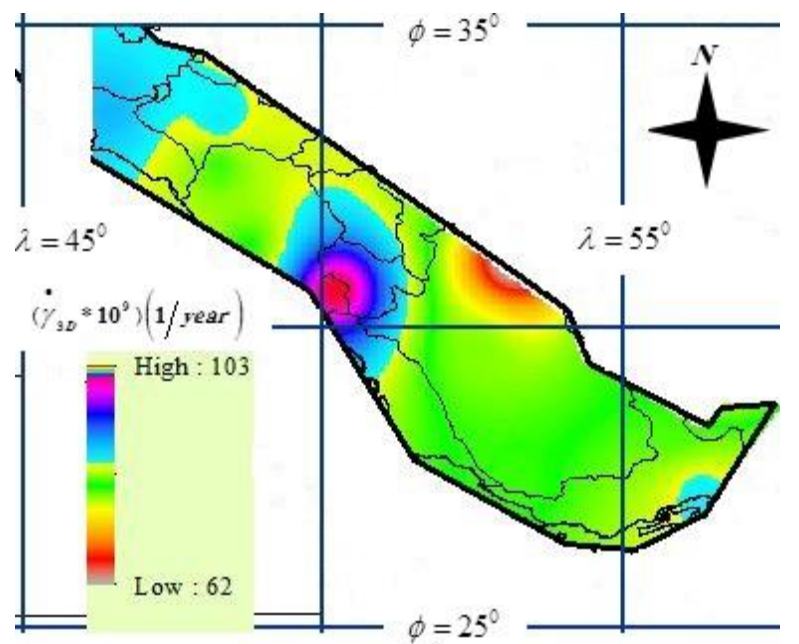

Figure 10. Changes of the 3D shear strain rate in Zagros

If we consider the density of the earthquakes larger than 5 Richter, we can describe the above results more clearly. Figure (11) shows the location of these earthquakes. 


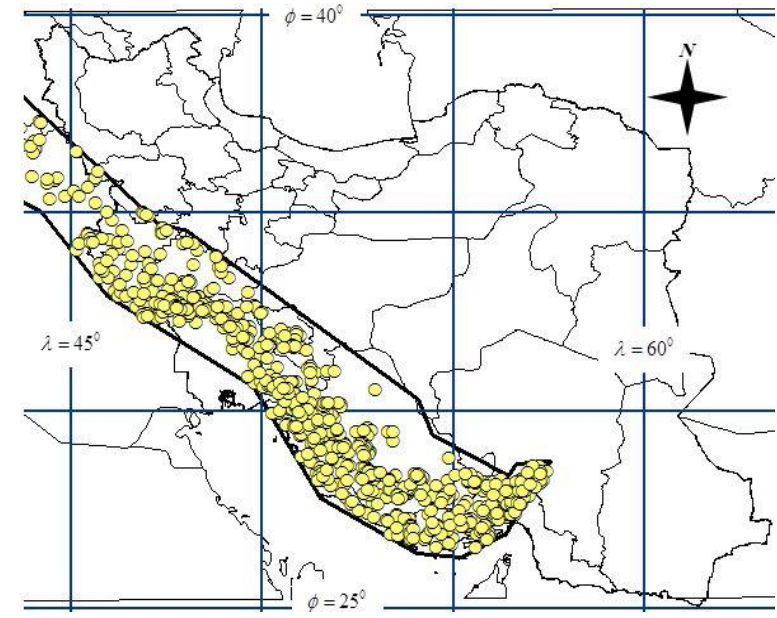

Figure 11. Density of the earthquakes with $M_{W} \geq 5$ in Zagros

\section{CONCLUSIONS}

Considering the results, we conclude that most of the surface deformation in Zagros have been created in an aseismic way and the earthquakes have had little contribution in making it. The studies carried out by (Jackson and McKenzie, 1988) revealed only $10 \%$ of the total deformation in Zagros was created by the earthquakes and most of the Zagros deformation is aseismic.

Waveform modelling shows that the large earthquakes in Zagros, happen in upper crust up to the depth of $15 \mathrm{~km}$ (Jackson and Fitch, 1981; Ni and Barazangi, 1986). Given that there are systematic errors in the depth measurements of earthquakes, the estimated seismogenic thickness shows a good coincidence with the results of waveform modelling. Investigating the density of the earthquakes larger than 5 Richter also shows that the major earthquakes occur in the southern Zagros and some parts of the central Zagros. The seismogenic thickness in these areas is lower than the other parts, and the information obtained from the 3D strain rate tensor also shows that the volumetric reduction of the province in these regions is slower than the other parts of Zagros.

According to the results, it is obvious if we had more permanent stations, we would be able to obtain more detailed information about the deformation rate.

\section{REFERENCES}

Baker, C., Jackson, J., \& Priestley, K. (1993). Earthquakes on the Kazerun line in the Zagros mountains of Iran: strike-slip faulting within a fold-and-thrust belt. Geophys.J.Int, 115, 41-61.

Berberian, M. (1995). Master "blind" thrust faults hidden under the Zagros folds: Active basement tectonics and surface morphotectonics. Tectonophysics, 193-224.

Drake, S. (2002). Converting GPS Coordinates to Navigation Coordinates. Edinburgh: DSTO Electronics and Surveillance Research Laboratory.

Hessami, K., Jamali, F., \& Tabassi, H. (2003). Major active faults of Iran, scale 1:2500000. Tehran: International Institute of Earthquake Engineering and Seismology.

Jackson, J., \& Fitch, T. (1981). Basement faulting and the focal depths of the larger earthquakes in the Zagros mountains (Iran). Geophys.J.R.Astron.Soc, 64, 561586.

Jackson, J., \& McKenzie, D. (1988). The relationship between plate motions and seismic moment tensors and the rates of active deformation in the Mediterranean and Middle East. Geophys.J.Int, 93, 45-73.

Jackson, J., Fitch, T., \& McKenzie, D. (1981). Active thrusting and the evolution of the Zagros fold belt thrust. Geological Society, 371-379.

Jekeli, C. (2006). Geometric Reference Systems in Geodesy. Ohio State University.

Kanamori, H. (1977). Seismic and aseismic slip along subduction zones and their tectonic implications. Maurice Ewing Ser, 1, 162-174.

Mirzaei, N., Gao, M.-T., \& Chen, Y.-T. (1997). A uniform catalog of earthquakes for seismic hazard assessment in Iran. ACTA SEISMOLOGICA SINICA, 10, 713726.

Mirzaei, N., Mengtan, G., \& Yuntai, C. (1998). Seismic source regionalization for seismic zoning of Iran: major seismotectonic provinces. J.Earthq.Pred.Res, 7, 465495.

Mostafazadeh, M., Alptekin, O., \& Oncel, A. (2000). Body waveform modeling of five moderately earthquakes in the Zagros fold thrust belt. J.S.E.E, 2, 1-10.

Mousavi-Bafrouei, S. H., Mirzaei, N., \& Shabani, E. (2014). A declustered earthquake catalog for the Iranian Plateau. ANNALS OF GEOPHYSICS, 57, 1-25.

Ni, J., \& Barazangi, M. (1986). Seismotectonics of Zagros continental collision zone and a comparison with the Himalayas. J.Geophy.Res, 91(B8), 8205-8218.

Scordilis, E. (2006, 1 12). Empirical global relations converting $\mathrm{Ms}$ and $\mathrm{mb}$ to moment magnitude. Journal of Seismology, 225-236.

Talebian, M., \& Jackson, J. (2004). A reappraisal of earthquake focal mechanisms and active shortening in the Zagros mountains of Iran. Geophysical Journal International, 156, 506-526.

Vernant, P., Nilforoushan, F., Hatzfeld, D., Abbassi, M., Vigny, C., Masson, F., et al. (2004). Present-day crustal deformation and plate kinematics in the Middle East constrained by GPS measurements in Iran and northern Oman. Geophys.J.Int, 157, 381-398.

Walpersdorf, A., Hatzfeld, D., Nankali, H., Tavakoli, F., Nilforoushan, F., Tatar, M., et al. (2006). Difference in the GPS deformation pattern of North and Central Zagros (Iran). Geophys.J.Int, 167, 1077-1088.

Ward, S. N. (1998, 1 21). On the consistency of earthquake moment rates, geological fault data, and space geodetic strain: the United States. Geophys. J. Int, 172-186.

Yenier, E., Erdogan, O., \& Akkar, S. (2008). EMPIRICAL RELATIONSHIPS FOR MAGNITUDE AND SOURCE-TO-SITE DISTANCE CONVERSIONS USING RECENTLY COMPILED TURKISH STRONG-GROUND MOTION DATABASE. The 14th World Conference on Earthquake Engineering. Beijing. 